\title{
Lipides polaires marins
}

Oléagineux, Corps Gras, Lipides. Volume 11, Numéro 2, 142-5, MARS/AVRIL 2004, Formulations et applications

Auteur(s) : Jacques FANNI, Michel LINDER, Michel PARMENTIER

ENSAIA, Laboratoire de Physicochimie et de Génie Alimentaires, 2, avenue de la Forêt de Haye, 54500 Vandouvre-lès-Nancy, France

Résumé : Les lipides polaires marins, notamment les phospholipides ( $\mathrm{PL}$ ), retiennent depuis quelques années l'attention des chercheurs et des industriels en raison de leur composition, particulièrement riche en acides gras polyinsaturés à longue chaîne (AGPI-LC). Ils combinent ainsi les propriétés reconnues des AGPI-LC à l'intérêt métabolique et structural des phospholipides. Les sources sont nombreuses et d'accès très diversifié. Le défi industriel provient de leurs caractéristiques amphiphiles et aromatiques particulièrement marquées qui rend leur extraction très difficile.

Summary : Due to the fact that they contain high amounts of long-chain polyunsaturated fatty acids (LC-PUFA), marine polar lipids (mainly phospholipids, PL), are subject of fundamental and industrial concern for few years. They combine the nutritional properties of LC-PUFA to the structural and metabolic aspects of phospholipids. Several and various potential sources are available. However, due to their amphiphilic and aromatic properties, industrial extraction still remains a challenge.

Mots-clés : lipide polaire marin, phospholipide, microalgue, krill, poisson

Keywords : marine polar lipid, phospholipid, micro-algae, krill, fish

\section{ARTICLE}

On connaît depuis maintenant plusieurs années les propriétés nutritionnelles des AGPI-LC, présents notamment dans les organismes marins. La littérature sur le sujet est aujourd'hui abondante. II était naturel de s'intéresser de plus près aux lipides polaires, lipides de structure par excellence, impliqués dans la constitution des membranes cellulaires et dans le fonctionnement du système nerveux. Les lipides polaires marins combinent donc a priori les propriétés reconnues des AGPI-LC à l'importance structurale et métabolique des phospholipides, pour ne parler que de ceux-ci. Dans l'article qui suit, et sans prétention exhaustive, nous avons pris le parti de détailler la composition des phospholipides des microalgues d'intérêt et de la faune à sang froid. Nous avons volontairement négligé les cétacés et les pinnipèdes dans la mesure où l'exploitation industrielle de ces animaux est condamnée à terme. Une énumération de la composition lipidique de leur chair en vue d'une exploitation industrielle n'eût été qu'anecdotique. 


\section{Les lipides polaires}

Contrairement aux lipides neutres qui participent directement au stockage énergétique, les lipides polaires, représentant 10 à $20 \%$ des lipides totaux, ont des fonctions essentiellement structurales et métaboliques, notamment dans les membranes cellulaires. On les classe habituellement de la manière suivante :

\section{Les phospholipides ou glycérophospholipides}

Le plus simple d'entre eux est l'acide phosphatidique correspondant à une molécule de sn-glycérol-3phosphate (anciennement acide L- $\alpha$-glycéro-phosphorique) où les fonctions alcools correspondant aux carbones 1 et 2 du glycérol sont estérifiées par des acides gras saturés (préférentiellement en sn1) ou insaturés (préférentiellement en $s n-2$ ). L'une des fonctions acides, demeurée libre dans le groupement phosphate, peut être estérifiée soit par un amino-alcool (sérine, éthanolamine ou choline) conduisant à la phosphatidylsérine (PS), la phosphatidyléthanolamine (PE) ou la phosphatidylcholine ( $\mathrm{PC}$ ), soit par un sucre (inositol, un inositol monophosphate ou diphosphate). Dans ce cas, il y a formation de phosphatidylinositol (PI), phosphatidylinositol monophosphate ou diphosphate.

\section{Les plasmalogènes}

Ils correspondent à des phosphatidyléthanolamines (ou cholines) dans lesquelles l'une des deux chaînes acyles est remplacée par une chaîne alkyle ou alkényle saturée ou insaturée.

\section{Les cardiolipides ou diphosphatidylglycérols}

Ils sont composés de deux molécules d'acide phosphatidique reliées au niveau de leurs groupements phosphates par une molécule de glycérol.

\section{Les sphingolipides}

Tous ces composés présentent dans leur structure une molécule de céramide résultant de l'association par liaison amide d'une molécule d'acide gras et d'une molécule de sphingosine. Selon le sphingolipide, la sphingosine du céramide est liée par sa fonction alcool primaire :

- soit à une molécule d'acide phosphorique, elle-même unie à une molécule de choline (sphingomyéline de la myéline ou des membranes des cellules animales);

- soit à une molécule d'hexose (glucose ou galactose), celle-ci pouvant être liée à une molécule d'hexosamine (galactosamine) ou à une ou plusieurs molécules d'hexoses (sphingoglycolipides).

Selon le nombre total de molécules d'hexoses et hexosamines, on distingue parmi les sphingoglycolipides, des céramides monohexosides (ou cérébrosides), des céramides di-, tri-, tétrahexosides.

Les cérébrosides peuvent avoir l'hydroxyle en position sn-3 du galactose estérifié par l'acide sulfurique (cérébrosides sulfates ou sulfatides). Les gangliosides (tissu nerveux, membranes des érythrocytes, etc.) sont des sphingoglycolipides dont la partie polyhexoside contient une ou plusieurs molécules d'acide sialique (ou acide neuraminique) en plus des hexoses et hexosamines. Ces lipides polaires contenant des oses sont fréquemment regroupés sous la terminologie générique 
de glycolipides. Alors que les phospholipides (PL) constituent la majeure partie des lipides polaires des membranes cellulaires, les glycolipides naturellement présents en faible quantité, occupent cependant une place très importante dans le tissu nerveux [1].

\section{Les lipides polaires marins}

Comme les triacylglycérols, les lipides polaires marins se caractérisent par leur richesse exceptionnelle en acides gras polyinsaturés, ce qui en fait une ressource potentielle très importante, car leur exploitation industrielle n'en est qu'à ses balbutiements. À l'origine de la chaîne alimentaire marine, les microalgues et la microfaune du plancton participent largement à la composition en acides gras que l'on rencontre chez les poissons. Naturellement, les coquillages font également partie de cette chaîne. Cependant, hors la coquille, ils ne génèrent pas de sous-produits, ce qui limite leur intérêt.

\section{Les microalgues}

Les microalgues peuvent contenir des quantités importantes de lipides dont la composition en acides gras ressemble à celle des plantes supérieures. Dans les lipides polaires, la proportion en acides gras polyinsaturés, notamment I'EPA (C20:5n-3) et le DHA (C22:6n-3) varient selon les espèces. Chez Isochrysis galbana, une microalgue largement cultivée aujourd'hui, le DHA est un acide gras majoritaire des phospholipides [2]. II en va de même chez de nombreux Dinoflagellées [3]. Les glycolipides n'en contiennent qu'une quantité réduite, mais c'est dans cette classe de lipides polaires que I'on retrouve la majeure partie de l'EPA chez Porphyridium purpureum [4] et Skeletonema costatum [5].

Chez la spiruline (S. platensis, S. maxima), une algue des eaux sodées de certains lacs tropicaux, célèbre pour sa capacité encore non démontrée à résoudre le déficit alimentaire de certaines populations, le contenu en lipides varie entre 5 et $11 \%$ selon les auteurs [6-9]. La fraction saponifiable ( $83 \%$ des lipides totaux) est principalement composée de monogalactosyl et digalactosyldiglycérols ( $23 \%$ ), de sulfoquinovosyldiglycérols (5\%). Le phosphatidylglycérol intervient pour $26 \%$ de cette fraction, ce qui est relativement élevé, alors que les phospholipides classiques (phosphatidylcholine, phosphatidyléthanolamine et phosphatidylinositol) ne se retrouvent pas en quantités appréciables. Curieusement, les triglycérides ne sont présents qu'à l'état de traces $(0,3 \%)$ et près de $5 \%$ des phospholipides ne sont pas définis. Par ailleurs, chez cette microalgue, les acides gras de la série $\omega 6$ se limitent aux acides linoléique ( 9 à $12 \%$ du total acides gras) et $\gamma$-linolénique (13 à $40 \%$ du total). II n'y a ni acides gras $\omega 3$, ni acides gras polyinsaturés à longue chaîne (AGPI-LC). On retrouvera ceux-ci, notamment I'EPA en quantité majoritaire, chez Porphyridium purpureum (anciennement cruentum) [10]. On peut ajouter que, plusieurs auteurs ont étudié la production d'EPA et d'acide arachidonique chez les Rhodophytes et les Phéophytes [11, 12].

\section{Le krill}

Le krill n'est pas à proprement parler une espèce, mais plutôt une famille de crustacés voisins de la crevette. II s'agit de l'un des premiers éléments de la chaîne alimentaire marine à laquelle on peut ajouter les copépodes. Présent en abondance dans tous les océans, notamment les mers les plus froides, il peut contenir une quantité très variable de lipides $(7,7$ à 27,1 \% chez Meganyctiphanes norvegica) dont $40 \%$ à $60 \%$ sont des phospholipides [13]. II s'agit donc d'une ressource potentielle 
de lipides polaires extrêmement importante. La PC est généralement le phospholipide majoritaire (environ $50 \%$ des PL totaux). Chez Euphausia superba, la PE est un phospholipide particulièrement riche en AGPI-LC [14-16]. II faut également souligner que cette teneur en PL est particulièrement élevée en période de reproduction, quand les femelles transportent leurs œufs entre leurs pattes abdominales. Les PL peuvent alors représenter jusqu'à $75 \%$ des lipides totaux.

\section{Les coquillages}

D'après King et al. [17], la teneur en lipides des bivalves peut atteindre 3,6\%, cependant, des valeurs de 1 à $2 \%$ sont plus courantes. II semble que le régime alimentaire ait une influence prépondérante sur la teneur en AGPI des lipides neutres et des phospholipides, notamment dans les gonades tant mâles que femelles. Ainsi, Soudant et al. [18] ont par exemple montré que la quantité d'EPA dans les PL est 3,6 fois plus élevée dans un groupe de Pecten maximus ayant reçu un régime algal à base de Chaetoceros calcitrans comparé à un autre groupe alimenté en Isochrysis galbana. Inversement, le DHA est 1,5 fois plus élevé dans le deuxième groupe. Cette influence doit toutefois être tempérée par le fait que le DHA et l'acide arachidonique s'accumulent nettement dans les PL indépendamment du régime. On a pu également noter que des altérations importantes surviennent dans les lipides neutres et surtout les $\mathrm{PL}$ de Patinopecten yessoensis au cours du stockage à $-20^{\circ} \mathrm{C}$. Etant donné l'amplitude de l'altération, notamment sur l'EPA et le DHA, les auteurs impliquent une lipolyse enzymatique endogène associée à une auto-oxydation [19].

\section{Les poissons}

Chez les poissons, la teneur et la composition lipidiques varient avec l'âge, le cycle sexuel et les facteurs environnementaux tels que la température et la salinité de l'eau [20,21]. Dans le groupe des poissons "gras", la teneur en lipides des muscles, essentiellement des triacylglycérols, peut varier de 1 à $25 \mathrm{~g} / 100 \mathrm{~g}$ de chair selon la saison et l'alimentation. Entre ces valeurs extrêmes, la masse moyenne de graisse est plutôt de l'ordre de $5 \%$ chez les poissons sauvages et de $15 \%$ chez les poissons d'élevage. Les di- et monoacylglycérols, principalement issus de l'hydrolyse des triacylglycérols au sein du muscle, ne représentent que des quantités négligeables et les phospholipides sont quasi absents de la masse musculaire. Chez les poissons " maigres ", les lipides sont surtout des triacylglycérols ( $35 \%$ des lipides totaux) associés à une forte proportion de phospholipides $(65 \%)$ intimement liés aux protéines, car ils sont constitutifs des membranes cellulaires [22]. Cette teneur apparemment élevée est trompeuse, car les poissons " maigres" contiennent généralement moins de $5 \%$ de graisse. En outre, les phospholipides sont plutôt concentrés dans les tissus cérébraux où ils peuvent représenter jusqu'à $65 \%$ de la masse humide. La phosphatidylcholine représente souvent plus de $50 \%$ des PL, suivie par la phosphatidyléthanolamine pour environ $30 \%$. Ils sont en général très riches en acides gras polyinsaturés à longue chaîne (AGPI-LC). Plusieurs auteurs ont montré que la teneur en PL reste sensiblement constante, quelle que soit la concentration en lipides neutres, ce qui paraît logique compte tenu de leur rôle essentiellement structural [23-25]. La proportion des différents acides gras varie en fonction de la fraction lipidique considérée. Dans le cas de nombreuses espèces méditerranéennes et des petits pélagiques (sardine, chinchard, maquereau), la composition en acides gras dans les PL et les lipides neutres est généralement identique. Cependant, la proportion d'acides gras monoinsaturés est supérieure dans les triacylglycérols, alors que celle des AGPI est plus élevée dans les PL. Les AGPI dans les PL peuvent ainsi représenter plus de $58 \%$ des acides gras de la 
fraction lipidique totale [26], avec l'EPA et le DHA dépassant fréquemment $50 \%$ des acides gras totaux dans les PL de la plupart des poissons. Le DHA est particulièrement abondant dans les PL musculaires des salmonidés et des calmars $[25,27]$. Des résultats similaires sont observés chez différentes espèces de thon et de calamar [28] (tableau 1).

Tableau 1. Pourcentage d'acides gras n-3 et n-6 en position sn-2 dans la classe des phospholipides de différentes espèces de thons [30]. Les valeurs entre parenthèses représentent le \% de DHA.

\begin{tabular}{|l|l|l|l|}
\hline Espèce de thon & AGPI n-3 & AGPI n-6 & n-3/n-6 \\
\hline (Terminologie anglo-saxonne) & & & \\
\hline Albacore & $58,3(47,3)$ & 9,3 & 6,3 \\
\hline Little tunny & $44,5(37,7)$ & 10,2 & 4,4 \\
\hline Skipjack & $52,8(46,0)$ & 10,5 & 5,0 \\
\hline Frigate & $61,0(51,4)$ & 9,3 & 6,6 \\
\hline Big eye tuna & $65,0(58,1)$ & 10,1 & 6,5 \\
\hline Yellowfin & $60,0(53,1)$ & 7,5 & 8,1 \\
\hline Bluefin & $61,8(53,7)$ & 9,0 & 6,9 \\
\hline Bonito & $60,4(50,5)$ & 5,8 & 10,4 \\
\hline
\end{tabular}

Pour la plupart des poissons de Méditerranée, notamment les petits pélagiques, la teneur en C22:6n3 dans les PL est plus élevée que celle de $C 20: 5 n-3$. On y trouve également très peu de $C 22: 1$ et C20:4n-6 [27]. En revanche, les triacylglycérols sont riches en C18:1, C20:1 et C22:1 et le rapport C22:6n-3/C20:5n-3 est plus faible que dans les phospholipides. De manière générale, les acides gras les plus insaturés ou à courte chaîne occupent préférentiellement la position sn-2. On a également déterminé que sur les $\mathrm{PL}$, les acides gras polyinsaturés à longue chaîne occupent principalement cette position, notamment chez le saumon et le thon. Cette position favorise notamment la fluidité membranaire, donc le transport de substances au travers des membranes cellulaires. C'est notamment le cas du phosphatidylinositol et de ses dérivés phosphorylés qui agissent au niveau des signaux de transduction [29]. Cette richesse particulièrement élevée en AGPI dans les phospholipides se fait au détriment des acides gras monoinsaturés (AGMI) lorsqu'on les compare aux triacylglycérols, alors que la proportion d'acides gras saturés (AGS) reste sensiblement constante. Dans la classe des lipides polaires, les AGPI présentent des chaînes carbonées plus longues et le rapport $\mathrm{C} 20+\mathrm{C} 22 / \mathrm{C} 18$ est de 4,3 à 9,8 fois supérieur à celui que l'on peut observer dans les lipides neutres. Chez le saumon, le DHA à lui seul représente 19,38\% des acides gras dans les phospholipides contre 9,58\% dans les triacylglycérols [24]. Parmi les AGPI présents dans les phospholipides totaux, c'est l'acide docosahexaénoïque (DHA) qui reste majoritaire dans la plupart des cas. Cette observation est appuyée indirectement par les 
travaux de Aubourg et al. [30] qui ont étudié la régio-distribution des AGPI des phospholipides chez le thon Albacore pendant la cuisson. Il est clair que les AGPI à longue chaîne (22:6n-3, 20:5n-3 et 20:4n-3) sont préférentiellement estérifiés sur la position sn-2 (tableau 2).

Tableau 2. Distribution des acides gras sur les positions sn-1 et sn-2 des phospholipides de thon Albacore avant et après cuisson (d'après [30]).

\begin{tabular}{|l|l|l|l|l|}
\hline \multicolumn{4}{|l|}{} & \multicolumn{2}{l|}{ Albacore frais } & Albacore cuit \\
\hline Acide gras & sn-1 & sn-2 & sn-1 & sn-2 \\
\hline C16 :0 & 20,8 & 6,7 & 22,3 & 7,9 \\
\hline C18 :0 & 43,1 & 2,1 & 34,2 & 2,2 \\
\hline C18 :1n-9 & 7,7 & 13,7 & 7,8 & 20,2 \\
\hline C20 :5n-3 & 1,2 & 9,3 & 2,0 & 8,2 \\
\hline C22 :6n-3 & 9,3 & 47,3 & 14,7 & 39,3 \\
\hline
\end{tabular}

Selon des travaux assez anciens [31, 32], cette distribution des AGPI-LC en sn-2 leur assurerait une protection contre l'oxydation durant les processus métaboliques. Cependant cette position est thermolabile, on observe notamment une diminution du DHA au profit de l'acide oléique. En même temps, l'acide stéarique diminue en sn-1 au profit du DHA et de l'acide arachidonique (C20:4n-6) [33].

Il est intéressant d'observer que la teneur et la composition de la graisse de saumon varient selon que le poisson est sauvage ou élevé en fermes aquacoles. Le saumon d'élevage accumule ainsi plus d'acides gras saturés que son confrère sauvage (il est d'ailleurs beaucoup plus gras que ce dernier). La teneur en AGPI dans les PL est notablement différente entre les deux groupes. Ainsi, le DHA est significativement plus élevé chez le saumon sauvage, alors que les PL du saumon d'élevage contiennent moins d'EPA. Ces résultats tendent à montrer que le DHA chez le saumon serait sous contrôle génétique et que la conversion des AGPI d'un rôle fonctionnel à un rôle nutritionnel serait plus élevée chez le saumon d'élevage [34]. Sachant que la majeure partie des PL est localisée dans le tissu cérébral, il peut paraître surprenant que les travaux sur la composition en PL du cerveau de poisson soient relativement rares. Les principales études et compilations bibliographiques ont été réalisées par des chercheurs russes de l'époque de I'URSS. Ainsi, l'un des articles les plus anciens se rapporte à la composition en acides gras des PL du cerveau, du foie et des muscles d'un saumon anadrome (Oncorhynchus nerka) au cours de différentes périodes et lieux de résidence du poisson [35]. Les auteurs distinguent ainsi une composition type "mer " et " rivière " dans le foie et les muscles, alors que les PL cérébraux demeurent sensiblement constants, probablement en raison de leur rôle structural dans les membranes cellulaires. Un groupe de 10 chercheurs (dont les précédents) s'est intéressé à la composition en PL et glycolipides chez les poissons de mer [36]. Ils observent que le cerveau de ces poissons contient entre 1 et $2 \mathrm{mg}$ de phosphore/g de poids humide, 
avec une composition en PL individuels identique chez les téléostéens, alors que la teneur en PC et PE est moindre chez les élasmobranches. La composition de chacun de ces PL ressemble à celle des vertébrés à sang chaud, mais tous sont plus riches en AGPI que ceux de ces mêmes vertébrés. Les cérébrosides vont de 1,1 à $4,9 \mathrm{mg} / \mathrm{g}$ de masse humide chez les téléostéens, alors que cette valeur est considérablement plus haute chez les requins. Chez le poisson Mylossoma aureum (un piranha herbivore), la teneur en DHA dans la phosphatidyléthanolamine est significativement plus élevée dans le cerveau que dans le foie [37]. Une autre revue bibliographique regroupant 43 références recoupe l'ensemble de ces observations sur toutes les familles de poissons [38].

\section{Conclusion}

Après cette revue non exhaustive de la composition en lipides polaires des organismes marins, il est clair que des sources potentielles d'exploitation industrielle existent. Les algues ont un rôle important à jouer, malgré leur faible teneur globale en lipides. Ceux-ci et notamment les lipides polaires, ont souvent des structures moléculaires originales dont les propriétés demandent encore à être vérifiées. Le krill est un excellent candidat, car sa teneur en phospholipides est très élevée, mais il importe de gérer la ressource avec attention. Les poissons constituent un énorme potentiel, cependant la qualité des lipides polaires obtenus est obérée jusqu'à aujourd'hui par un défaut rédhibitoire: I'odeur. En effet, l'une des particularités des phospholipides de poisson, est leur capacité à retenir les odeurs, ce qui d'un point de vue industriel est catastrophique. Cela est particulièrement vrai si les phospholipides sont destinés à des applications cosmétiques où la demande est forte, mais encore peu satisfaite. Le défi technologique majeur doit donc consister à extraire ces composés de la manière la plus efficace et la moins agressive possible pour l'environnement. Il est donc probable que les technologies douces associant des enzymes à des solvants ou additifs non toxiques ont un bel avenir. Toutefois, bien que des avancées importantes aient vu le jour au cours de ces dernières années, ce défi reste encore à relever.

\section{RÉFÉRENCES}

1. LOVE RM.The Chemical Biology of Fishes $1980 ; 2: 1968-77$.

2. LEBLOND JD, CHAPMAN PJ. Lipid class distribution of highly unsaturated long chain fatty acids in marine dinoflagellates. J Phycol $2000 ; 36: 1103-8$.

3. SUKENIK A, WAHNON R. Biochemical quality of marine unicellular algae with special emphasis on lipid composition. I. Isochrysis galbana. Aquaculture $1991 ; 97: 61-72$.

4. LOPEZ ALONSO D, BELARBI EH, RODRIGUEZ-RUIZ J, SEGURA Cl. Acyl lipids of three microalgae. Phytochemistry $1998 ; 47: 1473-81$.

5. BERGÉ JP, GOUYGOU JP, DUBACQ JP, DURAND P. Reassessment of lipid composition of the diatom, Skeletonema costatum. Phytochemistry $1995 ; 39$ : 1017-21.

6. UJARD EU, BRACO U, MAURON J, MOTTU F, NABHOLZ A, WUHRMANN JJ, CLÉMENT G. Composition and Nutritive Value of Blue Green Algae (Spirulina) and their Possible Use in Food Formulations. $3^{\text {rd }}$. International Congress of Food Science and Technology, Washington 1970. 
7. HALLEM JJ, PASSWATER RA, MINDELLEM. Spirulina. Keats Publishing, Inc. New Canaan, Connecticut 1981.

8. ANTILLAN C. Cultivation of Spirulina for human consumption and for animal feed. International Congress of Food Science and Technology. Madrid (Spain) September 1974.

9. UDSON BJF, KARIS IG. The Lipids of the Alga Spirulina. J Sci Fd Agric 1974 ; 25 : 759-63.

10. SUKENIK A, LIVNE A, ZMORA O, MOKADY S. Production of $\omega 3$ polyunsaturated fatty acids by unicellular algae from laboratory to commercial application. In Progress in Biotechnology of photoautotrophic microorganisms, $6: 6^{\text {th }}$ Intern Conf Appl Algol1993; September $6^{\text {th }}$ Czech Republic.

11. CHOU HN, CHIANG YM. Filamentous culture of seaweeds for the production of polyunsturated fatty acids. In : Progress in Biotechnology of photoautotrophic microorganisms $1993 ; 6: 6^{\text {th }}$ Intern. Conf. Appl. Algol. September $6^{\text {th }}$ Czech Republic.

12. RADMER WS. Products from algae grown in closed reactors. In : Progress in Biotechnology of photoautotrophic microorganisms $1993 ; 6: 6^{\text {th }}$ Intern Conf Appl Algol September $6^{\text {th }}$ Czech Republic.

13. ALBESSARD E, MAYZAUDAND P, CUZIN-ROUDY J. Variation of lipid classes among organs of the Northern krill Meganyctiphanes norvegica, with respect to reproduction. Comparative Biochem and Physiol 2001 ; Part A 129 : 373-90.

14. CHAPELLE S. Lipid composition of tissues of marine Crustaceans. Biochem System Ecol $1977 ; 5$ : 241-8.

15. CLARKE A. Lipid content and composition of the pink shrimp, Pandalus montagui (Leach) (Crustacea : Decapoda). J Exp Mar Biol Ecol 1979 ; 38 : 1-17.

16. CHAPELLE S, DANDRIFOSSE G, ZWINGELSTEIN G. Incorporation of (H3-) oleic acid into phospholipids of the crab Maja squinado. Wash J Biol $1982 ; 40$ : 90-7.

17. KING I, CHILDS MT, DORSETT C, OSTRANDER JG, MONSEN ER. Shellfish : Proximate composition, minerals fatty acids, and sterols. J Amer Diet Assoc $1990 ; 90:$ 677-85.

18. SOUDANT P, MARTY Y, MOAL A, ROBERT R, QUERE C, LE COZ JR, SAMAIN JF. Effect of food fatty acid and sterol quality on Pecten maximus gonad composition and reproduction process. Aquaculture 1996 ; 143 : 3161-378.

19. BO YOUNG J, OHSHIMA T, KOIZUMI C. Changes in molecular species compositions of glycerophospholipids in the adductor muscle of the giant scallop Patinopecten yessoensis during frozen storage. J Food Lipids 1999 ; 6 : 131-47.

20. CORRAZE G, KAUSHIK S. Les lipides des poissons marins et d'eau douce. OCL $1999 ; 6,1$ : 111-5.

21. CORDIER M, BRICHON G, WEBER JM, ZWINGELSTEIN G. Changes in the fatty acid composition of phospholipids in tissues of farmed sea bass (Dicentrarchus labrax) during an annual cycle. Roles of environmental temperature and salinity. Comparative Biochemistry and Physiology Part B :

Biochemistry and Molecular Biology 2002 ; 133, 3 : 281-8. 
22. BODY DR, VLIEG P. Distribution of the lipid classes and eicosapentaenoic (20:5) docosahexaenoic (22:6) acids in different sites in blue mackerel (Scomber australasicus) fillets. J Food Sci 1989 ; 54 : 569-72.

23. Ackman RG. Seafood lipids. In : Seafood Chemistry, Processing technology and quality. Shahidi, F. and Botta, J.R, Blackie Acad.and Professional, 1994 ; NY. 34-48.

24. SHEWFELT RL. Fish muscle lipolysis - A review. J of Food Biochem $1981 ; 5: 79-100$.

25. BANDARRA NM, BATISTA I, NUNES, ML, EMPIS, JM, CHRISTIE WW. Seasonal changes in lipid composition of sardine (Sardina pichardus). J Food Sci 1997 ; 62 : 40-2.

26. HENDERSON RJ, TOCHER DR. The lipid composition and biochemistry of freshwater fish. Progr Lipid Res $1987 ; 26: 281-347$.

27. PASSI S, CATAUDELLA S, DI MARCO P, DE SIMONE F, RASTRELLI L. Fatty acid composition and antioxidant levels in muscle tissue of different mediterranean species of fish and shellfish. $J$ of Agric And Food Chem $2002 ; 50: 7314-22$.

28. IGARASHI D, HAYASHI K, KISHIMURA H. Positional distribution of DHA and EPA in phosphatidylcholine and phosphatidylethanolamine from different tissues of squids. J Oleo Sci 2001 ; $50: 729-34$.

29. THOMAS LM, HOLUB BJ. Nutritional aspects of fats and oils. In Technological advances in improved and alternative sources of lipids. Pp 16-49. Eds BS Camel,Y Kakuda. Blackie Academic, Professional 1994.

30. AUBOURG SP, MEDINA I, PEREZ-MARTIN R. Polyunsaturated fatty acids in tuna phospholipids : distribution in the sn-2 location and changes during cooking. J Agric Food Chem $1996 ; 44$ : 585-9.

31. BROCKERHOFF H, YUKOWSKI M, HOYLE R, ACKMAN RG. Fatty acid distribution in lipids of marine plankton. J Fish Res Board Can $1964 ; 21$ : 1379-84.

32. BROCKERHOFF H, HOYLE R, HWANG P, LICHTFIELD C. Positional distribution of fatty acids in depot triglycerides of aquatic animals. Lipids $1967 ; 3: 24-9$.

33. AUBOURG SP, SOTELO CG, PEREZ-MARTIN R. Assessment of quality in frozen Sardine (Sardina pilchardus) by fluorescence detection. J Am Oil Chem Soc 1998 ; 75 : 575-80.

34. PENG J, LARONDELLE Y, PHAM D, ACKMAN RG, ROLLIN X. Polyunsaturated fatty acid profiles of whole body phospholipids and triacylglycerols in anadromous and landlocked Atlantic salmon (Salmo salar L.) fry. Comparative Biochemistry and Physiology Part B 2003 ; 134 : 335-48.

35. KREPS EM, CHEBOTAREVA MA, AKULIN VN. Fatty acid composition of brain and body phospholipids of the anadromous salmon, Oncorhynchus nerka, from fresh-water and marine habitat. Comparative Biochem and Physiol 1969 ; 31, 3 : 419-30. 
36. KREPS EM, AVROVA NF, CHEBOTAREVA MA, CHIRKOVSKAYA EV, KRASILNIKOVA VI, KRUGLOVA EE, LEVITINA MV, OBUKHOVA EL, POMAZANSKAYA LF, PRAVDINA NI, ZABELINSKII SA. Phospholipids and glycolipids in the brain of marine fish. Comparative Biochem and Physiol Part B : Biochem and Molecular Biology 1975 ; 52, 2 : 283 -92.

37. HENDERSON RJ, TILLMANNS MM, SARGENT JR. The lipid composition of two species of Serrasalmid fish in relation to dietary polyunsaturated fatty acids. J Fish Biol 1996 ; 48, 3 : 522-

38. EL-SHATTORY Y. Review on fish phospholipids. Die Nahrung 1979 ; 23,2 : 179-86. 